\title{
Articulación de miradas para una aproximación a la inclusión social de adolescentes infractores de ley. Teorías de redes sociales y de sistemas sociales
}

\section{Enzo Zamorano Brugueras}

Magíster en Antropología y Desarrollo, Universidad de Chile. Psicólogo, Universidad ARCIS.

ezamorano@sanbernardo.cl

Resumen

La legislación nacional intenta conciliar objetivos aparentemente contradictorios para población penal adolescente: la sanción de la conducta delictiva y el resguardo de los derechos que le asisten a los menores de edad. Se analizan en este documento las dificultades que este proceso presenta a la política pública en una sociedad compleja, caracterizada por la diferenciación funcional de sistemas, la masificación de la exclusión y la individualización de la construcción de biografías personales.

\section{Abstract}

The Chilean legislation attempts to reconcile apparently conflicting objectives for adolescent prison population: the sanction of criminal acts and the protection of his rights of the adolescents. Discussed in this document the difficulties that this process presents a public policy in a complex society characterized by the functional differentiation of systems, mass exclusion and individualisation of the construction of personal biographies.

Palabras Clave: redes sociales, sistemas sociales, adolescencia, delito, inclusión

Keywords: social networks, social systems, adolescence, crime, inclusion

\section{Introducción ${ }^{1}$}

\subsection{Centralidad de la temática del delito y la seguridad ciudadana}

La seguridad ciudadana y el delito son sin lugar a dudas temas prioritarios para la ciudadanía. La encuesta de seguridad ciudadana ENUSC presenta a la delincuencia ocupando el segundo lugar justo después de la pobreza como principales preocupaciones de la población (ENUSC, INE-CHILE 2007), el mismo estudio refiere que el $86.6 \%$ de los encuestados cree que la delincuencia aumento en Chile y un 42.2\% manifiesta la convicción de que en los próximos 12 meses será víctima de un delito.

Pese a no ser un fenómeno que pueda ser considerado como nuevo ${ }^{2}$, la seguridad pública y el delito -como tópico- presentan en la actualidad una centralidad particular. Muestra de ello es la convergencia de diferentes actores en torno a ella, los que en articulación generan un nuevo nivel de complejidad, causando efectos sobre diversos 
Articulación de miradas para una aproximación a la inclusión social de adolescentes infractores de ley.

Teorías de redes sociales y de sistemas sociales

subsistemas sociales, y de ahí a la sociedad en su conjunto (Torres, E. De la Puente, P.).

El sistema político, el sistema judicial, los sistemas de control (policías), los medios masivos de comunicación y la ciudadanía ${ }^{3}$ configuran lo que podríamos denominar un cluster de interacciones que dispone a esta centralidad. Como tópico, la Seguridad Ciudadana y el Delito son tematizados (Wolf, 1991) orientando de esta forma el accionar de estos sistemas mediante comunicaciones públicas derivadas de una selección contingente del tema. Este proceso es particularmente evidente en el sistema político, toda vez que las semánticas de la Seguridad Ciudadana -sin distinción de tendencias políticas- le sirven de eje $e^{4}$ para gran parte de sus programas.

Cada uno de estos actores de manera más o menos coordinada ha desarrollado acciones para aprehender el fenómeno; centralidad en las semánticas políticas, nuevas regulaciones legales, inéditas acciones policiales, incremento de la cobertura de los medios de comunicación masivos y redoblado interés por parte de la ciudadanía por la consolidación de estándares de calidad de vida (PNUD 2003, Pizarro 2009). Para ilustrar esta situación podemos observar el crecimiento vertiginoso de la representación del tema en los medios masivos de comunicación, hecho que por su magnitud no es posible sólo explicar a través del incremento de la comisión de hechos delictivos, sino más bien exige ser tratado como muestra de interacción, sinergia y creciente centralidad social de la temática.

\subsection{Delito cometido por adolescentes.}

El delito cometido por adolescentes ${ }^{5}$ ha obligado a considerar elementos novedosos en el proceso y la aplicación de leyes como la que da contexto a este documento, estos antecedentes están por ejemplo relacionados con todos los derechos y garantías que les son reconocidos a los adolescentes en la Constitución, en la Convención sobre los Derechos del Niño y en los demás instrumentos internacionales ratificados por Chile que se encuentran vigentes, situación que configura un contexto inédito en el desarrollo de cuerpos legales en nuestro país, toda vez que la ley de responsabilidad penal adolescente (LRPA) asegura por primera vez tanto la responsabilización penal de los delitos como la integración social del infractor ${ }^{6}$. De ahí el reconocimiento y la necesaria incorporación de elementos de rehabilitación y reinserción social aquí presentes.

Existen pocas mediciones internacionales de delito juvenil, y las existentes plantean dificultades metodológicas importantes a la hora de realizar comparaciones entre países. Aún considerando ese hecho y entendiendo estos indicadores como información preliminar, podemos afirmar con los datos disponibles que a nivel mundial los delitos cometidos por menores de edad tienen una representación importante en el total. Por ejemplo en las estadistas Inglesas de detenciones policiales del año 1999 el 23\% correspondía a menores de edad. Mientras que en Chile durante el mismo año la representación de menores de edad en detenciones realizadas por carabineros llegaba a $7.6 \%$. Cifra que se incrementa entre el año 2000 y el 2005, llegando a representar el $10.2 \%{ }^{7}$ del total de detenciones.

Es también notorio el aumento de la comisión de los considerados delitos de mayor connotación social (robo con fuerza, homicidio, violaciones, robo con violencia, hurto, lesiones) el que ha aumentado como motivo de detenciones desde 12.025 a 26.969 entre los años 1997 y $2003^{8}$, esto implica un crecimiento de más del $100 \%$ en un 
periodo no superior a los 6 años, demostrando también el aumento de la violencia vinculada a estos hechos.

\subsection{Ley de responsabilidad penal adolescente.}

El 8 de junio del año 2007 se pone en vigencia en Chile la ley de responsabilidad penal adolescente (LRPA), cuerpo legal que tiene como principal objetivo la regulación de la responsabilidad penal de los indivuos mayores de 14 y menores de 18 años, a quienes se aplicarán procedimientos y sanciones especiales, en plena armonía con lo dispuesto por la Convención Internacional sobre los Derechos del Niño.

La propuesta recoge el principio de responsabilidad, estimando que si bien el adolescente no puede ser tratado como adulto, si cabe exigirle una responsabilidad especial, adecuada a su carácter de sujeto en desarrollo. Las sanciones que establece esta iniciativa legal resultan de la declaración de responsabilidad por la realización de alguna de las infracciones a la ley penal que ella contempla y que corresponde al catálogo del Código Penal, con exclusión de la gran mayoría de las faltas.

Los autores refieren que este cuerpo legal extiende las garantías del debido proceso y de la legalidad penal a la población menor de edad, además de agregar resguardos adicionales para las personas en proceso de formación que a través de esto pueden enfrentar a la justicia en una posición a lo menos equivalente a la de un adulto y en lo posible en circunstancias más ventajosas.

Sus principales características dicen relación con; la búsqueda de soluciones alternativas que eviten recurrir a los procedimientos judiciales, respetando siempre plenamente los derechos humanos y las garantías legales. Este sistema privilegia la concentración del procedimiento, procurando que éste sea breve y ágil, atendidas las características de la percepción del tiempo por parte de los adolescentes y procura que las consecuencias jurídicas (sanciones, penas o medidas) también sean especiales o diferentes y se distingan por un marcado carácter socioeducativo que busca facilite la reinserción social del adolescente infractor.

Sus principales diferencias con la anterior práctica de aplicación de medidas legales a este grupo etáreo son aquellas vinculadas con la remoción del proceso de discernimiento (procedimiento que de tener resultados favorables permitía la aplicación de la ley considerando al imputado como adulto), la garantía de un sistema de justicia especializado en todas las fases del procedimiento, y durante el control de ejecución de la sanción, que aseguren la capacidad e idoneidad de los operadores del sistema, incluyendo la incorporación de la obligatoriedad de defensoría legal de los imputados, otorgando condiciones jurídicas para un debido proceso.

Este nuevo sistema encuentra sus principios rectores por una parte en la consideración del adolescente como un sujeto responsable de sus actos (adecuando esta responsabilidad a su carácter de individuo en desarrollo) y por otra parte en la incorporación por ley de los conceptos de rehabilitación y reinserción social, mas allá de la sola punición, lo que queda contenido en la propuesta de encontrar en la sanción una "intervención socioeducativa amplia y orientada a la plena integración social del adolescente" (Art. 17 y 20 de la LRPA, № 20.084).

Según estadísticas del año $2008 \mathbf{1 9 . 7 5 5}$ adolescentes entre 14 y 18 años fueron sujetos de intervención del Servicio Nacional del Menor (SENAME) en el área de Responsabilidad Penal Adolescente, es decir, tuvieron que cumplir algún tipo de 
medidas por infracción a la LRPA N²0.084. De ellos 823 lo hicieron cumpliendo penas privativas de libertad. El resto se divide en aquellos que cumplen medidas en medio semi-cerrado y que alcanzan a los 811 casos y los que ejecutan sus medidas en medio libre, llegando a sumar durante el periodo mencionado $\mathbf{1 8 . 1 2 1}$ adolescentes, representando el $\mathbf{7 1 \%}$ de los casos.

\section{Modernidad y exclusión-inclusión social}

El fenómeno de la exclusión social es un elemento central para intentar comprender las características de la delincuencia juvenil en nuestro país, y para observar el dilema que presenta la pretensión de inclusión social de menores infractores.

Consideramos que la exclusión es un proceso instalado a nivel global y que este se ha masificado y generalizado. Además aceptamos la premisa de que lejos de ser un problema en vías de solución, es uno que circula en la dirección contraria, es decir, que con el tiempo, los consabidos avances técnicos, y la ingente cantidad de riqueza de la que es posible la sociedad tenga disposición, los fenómenos de exclusión no hacen más que agudizarse.

\subsection{Las condiciones de la modernidad}

\subsection{1 ¿Como incluye la sociedad hoy?}

Siguiendo a Robles podemos afirmar que este proceso de inclusión no es sino un mecanismo de señalamiento, una operación para hacer visibles a los individuos como actores o como coparticipes en la comunicación de la sociedad (Luhmann, 1997:918633 En: Robles, 2006). La modernidad ha modificado este mecanismo de señalamiento, por ejemplo, ¿Cómo funcionaba en otros periodos históricos esta selección de inclusión a la comunicación de la sociedad? En muchos casos pertenecer a determinado grupo social o casta, o ser miembro de cierta institución educativa, aseguraba mecánicamente la inclusión en otros sistemas parciales como; trabajo, salud, religión o política, entregando expectativas de estabilidad a los individuos, de seguridad. Hoy no es posible mantener estas expectativas, ya que la modernidad cambia los criterios de inclusión, en un aspecto central: Estar incluido de un sistema parcial, no significa estar incluido en otro (Robles, 2006:96)

Cada sistema parcial presenta sus propios criterios de inclusión, los que no necesariamente responden a una lógica compartida, por lo tanto, tampoco pueden ser observadas con criterios de racionalidad, es decir, no es posible aplicar modelos racionales para revertir los efectos de la exclusión con pretensiones omniabarcadoras. Esperar lo anterior implicaría solo una dilación ad infinitud de la llamada "integración social" refiere Robles, porque como mencionamos, la inclusión en un sistema no significa la inclusión en otro (Robles, 2006). Lo anterior presenta grandes dificultades para las políticas públicas cuyos programas estén orientados a ese fin, debido en gran medida a que está implícito en la aplicación de estas estrategias de “integración" la premisa de inclusión total.

Tomemos como ejemplo las estrategias que se aplican a menores infractores como parte de las medidas contenidas en la Ley de Responsabilidad Adolescente. Una de las líneas de intervención es la re-escolarización y habilitación técnica como mejoramiento de las condiciones de empleabilidad. 
Las dificultades que se pueden encontrar aquí tienen que ver con:

- Pertinencia. En tanto las iniciativas que se apliquen a esta población estén alejadas de los intereses de los beneficiarios, poco sintonizadas con las necesidades del mercado laboral y por último sean ineficaces a la hora de insertarse en los puestos de trabajo, ya que las empresas tengan énfasis de inclusión distintos a la búsqueda de población juvenil con nula o escasa experiencia y además con antecedentes delictivos.

- Temporalidad. Ya que inevitablemente las intervenciones de la política pública llegan tarde a la experiencia de vida de los adolescentes infractores. Los que en la mayoría de los casos ya han hecho importantes decisiones en sus biografías individuales, muy difíciles de revertir al momento de instalarse las intervenciones del aparato público. La temporalidad tiene otro factor a considerar, ya que se produce en la experiencia de los adolescentes infractores un doble y paradojal fenómeno temporal; por un lado una maduración anticipada, la que se evidencia en la elección temprana de pareja, inicio precoz de consumo de sustancias e inserción anticipada al mundo del trabajo, entre otras. Y por otra parte una inmadurez psicológica, la que se expresa en dificultades para mantener relaciones afectivas significativas, métodos de resolución de conflictos de baja calidad (violencia) y un insuficiente grado de tolerancia a la frustración. Lo que hace más complejo aún seleccionar e implementar acciones de inclusión social con esta población.

- Expectativas. Ya que las iniciativas implementadas con esta población en su mayoría se derivan de expectativas erróneas, utilizando criterios racionales de "integración social" normativa. Del tipo; "si pongo a disposición de " $X$ " tal habilidad " $Y$ " entonces el mercado laboral debería incluirlo. Al estar estas expectativas mal sintonizadas con la realidad de los sistemas funcionales, las intervenciones que se puedan implementar caen irremediablemente bajo el peso de la contradicción.

Parecería entonces necesario revisar la excesiva centralidad en el individuo que muestran estas estrategias. Para incorporar puntos de vista sino sistémicos si de relaciones. Medidas de intervención que contengan y observen la dimensión sociocomunitaria de la problemática.

\subsubsection{Exclusión / I nclusión}

Si pensáramos en términos comparativos acerca de la robustez de las dinámicas que se generan en ambas zonas de la selección sistémica, es decir en la de la inclusión y la de su contraparte la exclusión, podríamos decir que del lado no actualizado, es decir, del lado de la exclusión, esta robustez pareciera férrea. Robles manifiesta que esta eficacia en la exclusión pareciera operar con un grado de precisión insólito y perverso, efecto que se potencia toda vez que la exclusión de un sistema parcial generalmente activa una cadena de exclusiones acumulativas, un verdadero efecto dominó. El individuo que es desvinculado de su trabajo, al cabo de un tiempo quedará excluido además del sistema de salud, a su vez el sistema económico responderá también con eficacia y precisión expulsando al individuo y exponiendo esa expulsión en su respectiva plataforma de comunicación (DICOM), quien informará a otros actores para actúen en concordancia. Por el contrario las dinámicas de inclusión no tenderán a generar tal grado de sinergia. Se dirá entonces que la inclusión es lábil improbable y restrictiva, mientras que la exclusión es altamente probable, estable y acumulativa. 
Ahora bien y pese a que no es posible asimilar como sinónimos inclusión y bienestar o exclusión y pobreza. No es menos cierto que como hemos establecido al producirse efectos dominó en el lado de la exclusión si sea más posible encontrar acumulación de exclusión en sectores sociales desfavorecidos. En palabras de Mascareño esta situación tiene particulares características en Latinoamérica toda vez que la periferia del capitalismo aún "mantiene altos niveles de estratificación" lo que introduciría una mayor desigualdad a la hora de intentar incluirse, ya que existiría una "duplicación de la desigualdad de inclusión que favorece a las posiciones superiores de la estratificación moderna y afecta a las inferiores (Mascareño, 2005) ${ }^{9}$.

En resumen, aquellos individuos ubicados en las posiciones inferiores de la estratificación moderna tendrán un handicap importante; la exclusión en la que se encuentran se les aparecerá como altamente probable, estable y acumulativa, y los sistemas funcionales que los incluyan tendrán un componente mixto, ya que compartirían escenario con exclusiones simultaneas, por ejemplo estar incluidos en el sistema educacional en su formato público no encontrarán futuras inclusiones ya que este sistema se encuentra desprestigiado y descargado de potencialidad de inclusión, esta dinámica no generará recursividad de inclusión.

\subsection{I ndividualización}

Individualización significa dependencia del mercado en todos los aspectos de la vida. (Beck, 1998). En esta frase esta contenida una de las situaciones que consideramos centrales para entender las condiciones del fenómeno de la delincuencia juvenil, no estableciendo relaciones causales sino mas bien contextualizando el escenario social en el que este fenómeno (como otros) se desarrolla.

En las sociedades modernas - referirá Beriajn- se produce una coexistencia (problemática) entre dos modernidades. La de la expansión de las opciones y la de la expansión de los riesgos. Ambas son indisociables. En esta aparente dinámica entre oportunidades y riesgos los individuos ganan en libertad, debido en gran medida a la disolución de los vínculos sociales tradicionales (familia, clase, religión, etc.). Este proceso de individualización significa que los sujetos son sacados de sus tradiciones y de la seguridad preestablecida y se ven compelidos a crear sus propias biografías. En este sentido individualización significa que la propia biografía queda al margen de pautas previas y abierta a situaciones en que cada cual ha de elegir cómo actuar. Disminuye el aspecto de opciones de vida realmente alternativas y aumenta el aspecto de biografías auto-producidas, al margen de alternativas (Beck, 1998).

La individualización aquí propuesta es una individualización institucionalizada, no es la atomización o el simple aislamiento, es decir, configura un individuo arrojado a su arbitrio pero profundamente necesitado de vincularse a nivel institucional, necesita de las instituciones, y en ese sentido las instituciones de la sociedad moderna están programadas para llevar a los individuos hacia la individualización obligándolos a desarrollar biografías privadas carentes de un mayor sentido de integración social o como dirá Beck, con un nuevo y paradójico sentido de integración social.

Así un adolescente o un joven se vuelve dependiente del mercado laboral y, por lo tanto, de la educación, dependiente del consumo, dependiente de las regulaciones y abastecimientos sociales, de las posibilidades y modas en el asesoramiento médico, psicológico y pedagógico (Beck, 1998). Le será posible hacer de su vida lo que disponga, pero estas potencialidades estarán supeditadas a las capacidades de las 
instituciones de sintonizar con sus múltiples y muchas veces disímiles (entre individuos) demandas.

Beck distingue tres dimensiones de individualización:

1.Disolución de las precedentes formas sociales históricas y de los vínculos en el sentido de dependencias en la subsistencia y dominio tradicionales. A esto lo denomina la dimensión de liberación.

2.Perdida de seguridades tradicionales en relación al saber hacer, creencias y normas orientativas. A esto le denomina la dimensión de desencanto.

3.Instalación de un nuevo tipo de cohesión social, a esto le denomina la dimensión de control o de integración, con lo cual el significado del concepto se convierte precisamente en su contrario, es decir, que mientras este nuevo tipo de integración esta en funcionamiento no cumple su función integradora sino mas bien una función de individualización.

En la dimensión de liberación los adolescentes pueden disponer con mayor discrecionalidad que nunca de sus propias existencias, seteando los cursos que mejor les parezcan. Tendrán que responder eso si por las opciones que tomen, por ejemplo, en temas formativos -¿Que estudio?, $O$ en los casos de condiciones sociales deprivadas, ¿Para qué sigo estudiando?. Lo mismo con la profesión, el trabajo, el lugar de residencia, la elección de pareja o la cantidad de hijos, todas opciones que -en especial en niveles socioeconómicos bajos- aparecen recién esta generación, ya que sus padres no tuvieron que responder a ellas o al menos tuvieron relativa facilidad comparativa para responder, debido en gran medida a las condicionantes sociales que disminuían sus opciones.

No es poco esperable entonces observar la ansiedad que les produce el futuro a los adolescentes, como algo que verdaderamente no existe, porque realmente podría pasar cualquier cosa (contingencia), es decir, no sucederá necesariamente lo que ha sucedido (con mis padres). Esto -podríamos hipotetizar- estaría reflejado en la excesiva inmediatez que demuestran los jóvenes en general y los infractores en particular, la dimensión de desencanto.

Es así como en este escenario un adolescente infractor debe -al menos a nivel de conciencia- responder por sus actos partiendo desde si y culminando en si, doblando la responsabilidad individual, ya que el delito y en especial el delito como forma de vida, podría ser explicado cada vez mas como opción personal, y cada vez menos como una consecuencia de condicionantes sociales como en las teorías de la criminalización, con lo cual -creemos- se refuerza la identidad delictiva, la identificación uno a uno entre el delito y el individuo, expresado en la frase "es mi vida, es mi opción".

Los sujetos quedan entonces enfrentados a lo que los autores denominan oportunidades "riesgosas", en la medida que las mismas condiciones (el aumento de las opciones individuales) que les ofrecen potencialidades de libertad, también demuestran sus incapacidades de poder usar estas posibilidades. Son riesgosas estas posibilidades de libertad en la medida que no todos los sujetos tienen las mismas condiciones para enfrentar de manera óptima estos espacios de libertad. Si antes no tenía lo que necesitaba, el adolescente de nivel socioeconómico bajo podía recurrir a la explicación de clase, la "pertenencia" a una clase social "oprimida, alejada de los bienes por otra clase social opresora y depredante". Frente a la misma necesidad hoy, 
solo puede buscar las explicaciones en su propia biografía. Aquí la doble faz de estas oportunidades, por un lado los sujetos quedan "liberados" de las estructuras que los soldaban a sus auto-descripciones; por ejemplo como "pobres", para abrirse a las múltiples posibilidades que ofrece por ejemplo el mercado, las que se entiende están ahí para ser tomadas por cualquiera. Pero por otra parte este adolescente carecerá de los marcos explicativos que otorgaban las adscripciones a grandes grupos, los sentidos que estos grupos otorgaban a la red social y la conciencia de necesitar de sistemas funcionales para el logro de su objetivos, y así enfrentar el reto que le propone esta libertad (Dimensión de I ntegración).

\subsection{I ndividuación}

\section{Del "haz de tu vida lo que quieras" al "haz de tu vida lo que puedas"}

Como hemos establecido el individuo individualizado tiene una dependencia enorme de las instituciones, de los sistemas funcionales podríamos decir. Para disponer de su biografía con discrecionalidad el individuo debe sortear con éxito la masificación de la exclusión, la precarización de las relaciones laborales, la relativización de las relaciones afectivas, los cambios de estatus marital, etc. Para ello necesita un sistema social que le procure estabilidad, un paliativo a la dinámica exclusión-inclusión.

En una investigación desarrollada en Suiza ${ }^{10}$, se observo la relación existente entre "familia disociada" y "delincuencia". Bajo la premisa que se establece entre familias disociadas (monoparentales) y delincuencia adolescente. Los resultados mostraron falta de correlación entre tipos de familia (con ambos padres presentes - solo uno) y comisión de delitos por los miembros adolescentes, al respecto el autor reconoce el gran valor que tiene el sistema social como amortiguador de las contingencias individuales y familiares, “... la escasa diferencia entre los jóvenes de ambos tipos de familias se explican porque, en regla general, el sistema de seguridad social suizo permite que las familias disociadas desempeñen su tarea de socialización con la misma eficacia que las familias tradicionales". Las preguntas que caben son:

¿Que sucederá en sociedades en las que los sistemas de protección social no tengan la misma eficacia frente a la realidad de individuos individuales?

¿Qué podrán hacer con su libertad individuos que no disponen de la pertinencia y eficacia de soportes de seguridad social?

Hemos establecido que esta libertad es autoconfrontación en la medida en que el individuo debe volver sobre si para confrontar sus propias demandas, en el caso de la individualización euro-céntrica esta autoconfrontación es un proceso asistido por un estado que amortigua, mientras que en la realidad de países de la periferia del capitalismo mundial la individualización se trata de una autoconfrontación desregulada (Robles 2005) sin un estado que sirva de "colchón", lo que significa un aumento significativo de las inseguridades ontológicas. Es decir, que mientras que por un lado los procesos de modernización reflexiva ofrecen potencialidades individuales emancipatorias, por otro expone a los individualizados a mayor inseguridad. Para dar cuenta de esta diferencia Robles propone el uso del concepto de individuación.

Esto en el caso de los adolescentes infractores ofrece condiciones de análisis, toda vez que en tanto sujetos individuados están expuestos a estas "libertades riesgosas" las que en muchos casos solo acentúan el riesgo, obligándolos a depender de lazos 
sociales de solidaridad, los que a juicio nuestro en muchos casos vienen aparejados con las conductas disociales.

\subsection{Redes Sociales / Capital social}

\section{La perspectiva relacional para observar la exclusión.}

Como una forma de contrarrestar o compensar los efectos de la individualización, los jóvenes establecen grupos de pares, generando en muchos casos subculturas o submundos en los que sea posible establecer formas de relación distintas a las de la sociedad en su conjunto (Beck, 2000). Estos grupos son particularmente observables con referencia a la temática que convoca este artículo, dado que es indiscutible el peso que tiene en la conducta delictiva la influencia de los grupos sociales, tanto de pares como comunitarios en general. (Sutherland, 1970. Cohen 1955. Matza, 1964)

El enfoque teórico de las redes sociales intenta establecer status y roles a partir del análisis de las relaciones entre individuos sin imponer categorías a-priori o características de los actores (White et al. 1976 en: Espinoza 1995) en ese sentido se distancia de las particularidades individuales y centra su atención en la perspectiva relacional (Licha, 2002).

Estas relaciones están definidas por un criterio subyacente, lo que permite identificar estructuras sociales que generalmente no están formalmente definidas por la sociedad, el interés esta centrado en la forma en que las relaciones están ordenadas. En palabras de Garrido las estructuras "se convierten en un objeto de estudio central pues se postula que tal estructura es un condicionante de la formación de interés colectivo, de la definición de objetivos, de la constitución de las organizaciones y alianzas..." (Garrido, 2001) y ya que las estructuras se corresponden mas bien con las pautas de relaciones recurrentes en un grupo, antes que con agrupaciones de individuos con características similares, (Espinoza, 1995), este enfoque puede servir como medio de contraste a través del cual mirar lo velado, lo micro-social sin la necesidad de anteponer las demandas que impone la aplicación de la ley ni la estreches de la ideología. Poder mirar las relaciones que se dan en la exclusión sin la necesidad de volver sujeto de nuestro análisis al individuo y su circunstancia, de alguna forma librándose de la recursividad del sistema de la exclusión.

Como enfoque teórico el de las redes sociales se aproxima al par inclusión/exclusión desde los contenidos y cualidades de las relaciones de red. Observando estos contenidos y cualidades asociados a la condición de exclusión. Existen en este sentido importantes avances en materia de pobreza y las redes sociales que surgen para superarla (Espinoza, 1995).

En ese sentido centrarnos en los contenidos y cualidades de las relaciones de red nos permitiría una visión más completa de las relaciones interaccionales que el adolescente describe en procura del balance que las carencias que hemos hipotetizado presenta, en tanto individuo expuesto a una autoconfrontación desregulada, precariamente incluido en sistemas funcionales. Por ejemplo creemos que el comportamiento de los jóvenes se explicará mejor por las redes en las que se integra que por sus atributos de edad o genero.

El comportamiento de los individuos y grupos sus normas y valores está asociados a la posición ocupada en el sistema estructurado de relaciones sociales. No se niega la existencia y fuerza de las normas pero se estima que éstas únicamente operan dentro 
Articulación de miradas para una aproximación a la inclusión social de adolescentes infractores de ley.

Teorías de redes sociales y de sistemas sociales

de oportunidades y constricciones que son el resultado de la estructura de relaciones (Garrido, 2001)

\subsection{Tipos de I nclusión}

Utilizaremos las distinciones de inclusión primaria y secundaria para dar cuenta de la circularidad de estas operaciones, en tanto observamos que los esfuerzos contenidos en las actuaciones de la ley 20.084 se centran en facilitar inclusiones primarias, manteniendo aún cerrado el acceso a las redes de influencia y reciprocidad. Robles llamará a este suceso de integración; exclusión en la inclusión, definiéndola como "la inclusión que no es..." cuando no se tiene acceso a las redes de favores, influencias y reciprocidades de conveniencia (Schulze 1995 en: Robles 2005).

Es precisamente frente a estas distinciones que el enfoque de redes a través de la propuesta de Granovetter parece más pertinente. Ya que serían los lazos débiles los que aseguren la integración social a una escala mayor (Granovetter 1973 en: Espinoza 1995). Formando puentes entre redes que de otra forma estarían aisladas (Granovetter 1982). En alguna manera devolviendo a los niveles micro, a las relaciones interacciónales o diádicas la posibilidad creciente de volver sus interacciones en alguna medida recíprocas ${ }^{11}$.

\subsection{Ley de responsabilidad penal, políticas pública e integración social}

Hemos intentado establecer las condiciones que se presentan con referencia al delito adolescente, las condicionantes sociales que dan contexto al fenómeno, las expectativas que se establecen en función de la LRPA 20.084, y las dificultades que observamos se manifiestan especialmente con referencia a la pretensión de inclusión social de la mencionada ley.

Las políticas públicas insisten en presentar escenarios en los que es posible coordinar a los subsistemas de la modernidad para los fines que considera necesarios. Las semánticas del "crecimiento con igualdad" y de la "equidad", características de los gobiernos de la concertación, son pretensiones normativas lanzadas desde un ideal de inclusión.

Es importante volver sobre la distinción planteada con anterioridad, inclusión no es sinónimo de bienestar ni exclusión sinónimo de pobreza. Si la pretensión de la ley de Responsabilidad Adolescente busca incluir a estos individuos debe observar factores distintos a las condiciones que el individuo tenga o no para ser seleccionado por los subsistemas sociales. Debe necesariamente tomar distancia y observar estructuras de relaciones.

Utilizaremos las distinciones de inclusión primaria y secundaria para dar cuenta de la circularidad de estas operaciones, en tanto observamos que los esfuerzos contenidos en las actuaciones de la ley 20.084 se centran en facilitar inclusiones primarias, manteniendo aún cerrado el acceso a las redes de influencia y reciprocidad. Robles llamará a este suceso de integración; exclusión en la inclusión, definiéndola como "la inclusión que no es..." cuando no se tiene acceso a las redes de favores, influencias y reciprocidades de conveniencia (Schulze 1995 en: Robles 2005).

Creemos que las acciones contenidas en las estrategias de intervención de la LRPA fallan en encontrar y utilizar las potencialidades de estas redes de interacción, debido a 
su especial énfasis en las prestaciones individuales y a la búsqueda de integración normativa mediante la entrega de servicios de sistemas funcionales que llegan irremediablemente desfasados de la realidad de este sujeto infractor.

En virtud de lo anterior se considerará como fundamental en el proceso de selección de conductas prosociales de los adolescentes que han determinado utilizar medios delictivos para la consecución de sus biografías individuales, las características de estas redes de interacción y autoayuda, los tipos de recursos que sean capaces de distribuir y la densidad relacional que exista entre sus componentes.

Creemos que en la medida que los indicadores de redundancia y homogeneidad de los componentes de la red sean más bajos menor será también la incidencia de la comisión de delito. En otras palabras, creemos que en la medida de que las redes sociales de estos jóvenes sea más diversa, heterogénea y dispersa, menor será la posibilidad de estos de reincidir en conductas delictivas.

\section{Acerca de los resultados. Redes sociales e inclusión social de menores infractores en una sociedad compleja.}

En función de los hallazgos realizados en el proceso de investigación y en consonancia a las opciones teóricas y metodológicas utilizadas, es posible arribar a ciertas conclusiones. Primero se exponen las conclusiones referentes a las principales características de las redes de adolescentes infractores, posterior a ello se presentan los factores protectores encontrados en las redes de adolescentes no infractores para luego abordar los efectos que los procesos de individuación muestran en los adolescentes investigados, Por último se observan los roles que las redes sociales en general y las redes familiares en particular juegan en tanto estrategias de análisis e intervención en el proceso de inclusión.

Derivadas de estas conclusiones se anexan sugerencias a las estrategias que se implementan con población juvenil en situación de exclusión en general, y con población adolescente infractora en particular.

\subsection{Acerca de las redes sociales analizadas. Diferencias y principales características}

Es posible indicar diferencias significativas entre las redes de adolescentes infractores y las de aquellos que no han cometido delito. A continuación se presentan las características de las redes familiares, institucionales y de pares de adolescentes infractores.

\section{Red familiar de adolescentes infractores}

Los adolescentes infractores al ser consultados por su red familiar comienzan con una distinción decidora; mencionan solo a las personas que conforman el hogar ${ }^{\mathbf{1 2}}$, En ningún caso se mencionan vínculos familiares no presenciales, los que denominaremos su familia extendida pese a que estos fueran consanguíneos.

En tanto red familiar son numéricamente pequeñas (entre 5 o 6 vínculos) y particularmente compactas, con un grado de densidad relacional muy alto, en la que todos los vínculos se relacionan frecuente y cotidianamente. 
Articulación de miradas para una aproximación a la inclusión social de adolescentes infractores de ley.

Teorías de redes sociales y de sistemas sociales

Presentan en su mayoría un formato de familia reconstituida (padrastros), con evidentes dificultades para generar una imagen o discurso que culmine con éxito esta reorganización, en otras palabras permaneciendo en una constante espera por la identidad familiar.

El repertorio de recursos y alternativas que tienen a disposición para resolver problemas cotidianos presenta baja variabilidad. Al interior de la red se distribuyen recursos poco novedosos, volviendo sobre datos y soluciones prototípicos, aún cuando estos demuestren poca efectividad.

En cuanto a los roles que cada miembro juega al interior de la red; Padres, hijos, hermanos, pudo ser observado una "des-diferenciación funcional" alta. Son redes homogéneas, funcionando al estilo de "grupo de pares". Los roles o jerarquías tradicionales, parecen desdibujados. Los padres están imposibilitados para mostrar un camino orientando las acciones de los miembros en proceso de formación, presentan serias dificultades para proveer de los recursos materiales necesarios, se muestran impotentes a la hora de establecer límites a la conducta. Los hijos no están supuestos a obedecer, no tienen la obligación de cumplir deberes, no regulan su conducta en función de las expectativas de la normatividad. En este sentido parece pertinente manifestar que de manera paradójica la conflictividad al interior de este tipo de red familiar es baja, al no existir normas que transgredir o al ser estas estériles, no llegan a provocar conflicto. La cotidianidad transita en una calma contradictoria, llena de amenazas e incertidumbres y simultáneamente estable y rutinaria.

Conviven en su interior características al parecer contradictorias, ya que como mencionamos pese a mantener relaciones de una densidad relacional alta, esto no logra consolidar vínculos firmes y de confianza.

La novela familiar aparece marcada por el conflicto, situación que comparten con las redes familiares de adolescentes no-infractores, pero que a diferencia de estos no resuelven de buena forma. El conflicto aparece como irreversible, siempre motivo de rupturas definitivas, lo que viene en directo desmedro del aumento de posibilidades de variabilidad de recursos. En los relatos de los adolescentes el constante corte de relaciones con su familia extendida se extrema hasta casi hacerlos volver sobre si mismos, reducir sus contactos de confianza, a unos pocos miembros de esta red familiar, cada vez mas próxima, cada vez más pequeña.

Es frecuente la presencia de miembros infractores de ley, muchos de los cuales están cumpliendo medidas privativas de libertad. El rol de este miembro es central, en tanto encarna la ambigüedad propia de la conducta delictiva, por un lado es la vergüenza, quien peor lo hizo, el lugar que ninguno de los otros miembros de la red quiere ocupar. Y a la vez es el depositario de la admiración, del orgullo familiar, un mártir que sufre penalidades en beneficio de una red familiar y de una comunidad que ha optado por un estilo de inclusión alternativo que utiliza y valida las conductas antisociales, que tiene su precio, pero que quien lo paga gana su redención.

Por último es posible observar que pese a que los entrevistados no tienen la expectativa de recibir de sus redes familiares lo que ellos consideran los recursos "necesarios" para su normal desenvolvimiento, mantienen la expectativa de obtener lo que perciben que necesitan. Situación que en la red de no infractores se resuelve desistiendo o intercambiando los satisfactores, por otros susceptibles de ser obtenidos. 
La red familiar del infractor aparece impotente para colaborar con el adolescente en este proceso de negociación.

\section{Red de pares adolescentes infractores.}

Contrario a las imágenes más concordantes con el sentido común y la tradición, fue posible mediante esta investigación establecer que el adolescente infractor presenta dificultades para establecer vínculos de colaboración que trasciendan la inmediatez de la consecución de un satisfactor. Lo anterior pone entre paréntesis la caricatura del delincuente pandillero, asociado. No descartamos - por su puesto- la realidad de que muchas de las acciones delictivas se realicen en compañía o de manera concertada, pero no es menos cierto que estas colaboraciones cada vez menos dan cuentan de relaciones de confianza, sino mas bien de mutua conveniencia acotada y temporal. Lo anterior creemos abre una línea de reflexión en términos de las particularidades que la delincuencia juvenil presente en nuestro país.

Al ser consultados por sus redes de pares los adolescentes establecieron una distinción inicial para orientar sus respuestas, "existen amigos y amigos-amigos" dando cuenta de que no todos aquellos pares con los cuales compartían sus actividades eran sujetos de su entera confianza. Es así que establecen redes de 2 o 3 vínculos de "amigosamigos", circundada por una red de "amigos" algo mayor. El bien escaso en este tipo de relaciones es sin lugar a dudas la confianza, en este sentido nuevamente vuelve aparecer el conflicto y la rigidez para enfrentarlo como tema central. En este tipo de redes cada situación conflictiva tiene por acápite la ruptura del lazo, a diferencia de las redes de pares de no-infractores, las que pese a no estar totalmente desconflictuadas si presentan más ductilidad a la hora de enfrentar el conflicto, pudiendo ya sea reparar el vínculo conflictuado o reponerlo con uno nuevo.

Los vínculos de las redes de pares infractores son territorialmente cercanos, de hecho están mediados por el territorio, por las inmediaciones. Son los mismos amigos de la infancia, poco cambia o se moviliza. En este sentido demuestran muchas dificultades estableciendo nuevos vínculos con pares en espacios institucionales, en los que las relaciones deben comenzar desde otros parámetros, bajo otros códigos, muchas veces basados en habilidades distintas a las favorecidas en espacios informales, por ejemplo las relaciones que se establecen en el colegio pueden comenzar desde estándares cognitivos o intelectuales que podrían no ser el ambiente que más le acomode.

\section{Red institucional adolescentes infractores.}

Las redes institucionales en ambos casos (Infractores y no-infractores) son las que menos incidencia tienen en la experiencia vital de los adolescentes. Lo que podría interpretarse como una cierta desalineación entre las necesidades y expectativas de la etapa vital y la oferta que ponen a disposición estas instituciones.

Es particularmente agudo el caso en los adolescentes infractores, donde las intervenciones institucionales se ofrecen precarias, débiles, tercerizadas, poco pertinentes y evidentemente desfasadas. En respuesta a este tipo de oferta, los vínculos que los adolescentes establecen replican esta inestabilidad y precariedad. Y en los casos en que esta situación logra revertirse es debido a que los adolescentes logran generar vínculos directamente con los individuos que prestan los servicios, situación que se explica en gran medida por características de los propios individuos ejecutores, tales como; compromiso, entrega o simple carisma personal. Todas características 
Articulación de miradas para una aproximación a la inclusión social de adolescentes infractores de ley.

Teorías de redes sociales y de sistemas sociales

muy loables pero que no hacen sino evidenciar la falta de pertinencia en el diseño de las acciones que se desarrollan con esta población.

\subsection{Factores protectores presentes en las redes sociales de adolescentes no infractores.}

Es posible determinar ciertas características en las redes sociales de adolescentes no infractores susceptibles de ser consideradas factores protectores frente a las condicionantes sociales que enfrentan.

Si consideramos primero la composición de estas redes estas podemos advertir una diferenciación interna de roles y funciones clara. Existen jerarquías y límites observables, que funcionan e indican expectativas a la conducta de cada miembro de la red familiar. Los padres pueden - de buena o no tan buena forma- cumplir con el rol de proveedores, con los roles simbólicos de autoridad, y de continencia afectiva. Los hijos, mantienen su relación de verticalidad, aun cuando se viva agresiva o incómodamente. Este hecho pese a ser considerado por los adolescentes como una limitante a su autonomía es simultáneamente calificado como un soporte a su desarrollo.

Lo anterior configura situaciones conflictivas que tensan las relaciones entre los miembros de la red, sin embargo, es aquí donde estas redes demuestran otro de los factores de protección; su ductilidad. Tendiendo los conflictos a agotarse bajo la cotidianidad o a resolverse, pero no a generar quiebres en los vínculos, los que se mantienen y reparan, esto favorece a la red en tanto al mantener mayor cantidad de vínculos aumenta su nivel de variabilidad en el repertorio de respuestas posibles a situaciones complejas. Se podría decir que cada vez que la red es estresada por estas situaciones conflictivas y no cede sino se readecua "aprende", y por lo tanto está mas preparada para enfrentar nuevas demandas del entorno.

Otro factor de protección es la inclusión dentro de esta red familiar de vínculos de la familia extendida quien se visualiza como recursos utilizables. Los puentes entre estos adolescentes y el resto de su red familiar parecen más dispuestos a la conexión. Presentando mayor frecuencia de vínculos desterritorializados, familiares lejanos, viviendo en distintos lugares de la ciudad e incluso del país.

Por último un contrapunto entre la red de infractores y la de no-infractores fue una mayor presencia institucional. Las instituciones en esta red aparecen como agentes distribuidores de recursos; información, beneficios, soporte afectivo, posibilidades de conocer nuevas personas. En definitiva una puerta que lleva hasta nuevos bienes.

\subsection{Efectos de la individuación y la auto-confrontación}

En la medida en que los jóvenes quedan cada vez más separados de los lazos sociales por la progresiva individualización, las formas de percepción se vuelven cada vez menos sociales y cada vez más privadas y a-históricas. Siguiendo a Beck, “los hijos ignoran las situaciones de los padres, por no hablar ya de la de los abuelos. Es decir, el horizonte temporal de la percepción de la vida se reduce cada vez más, hasta encogerse finalmente en el caso límite de la historia del (eterno) presente, y todo gira en torno al propio yo de la propia vida" (Beck, 1998). 
Producto de esta tendencia hacia la individuación el adolescente queda desligado de sus lazos tradicionales, de las precedentes formas sociales históricas y de los vínculos en el sentido de dependencias en la subsistencia y dominio tradicionales.

Es posible observar en las redes sociales de adolescentes infractores una suerte de contracción. El adolescente infractor pierde confianza en la capacidad que tengan sus vínculos para procurarle recursos de todo tipo, con lo que cualquier esfuerzo por mantener estos vínculos parece carente de sentido, de esta forma el adolescente se ve obligado a volver sobre si mismo, su red colapsa sobre el ego, se reduce su capacidad de variabilidad de respuesta, se estandarizan y ritualizan las formas de pensar, se estrecha el mundo de posibilidades, se vuelve sobre las conductas mecánicas y puramente pragmáticas, el delito tiende a tener a cobrar -bajo estas condicionesevidente sentido.

Lo anterior además de marcar un cambio en la manera en que el delito juvenil se concebía históricamente en nuestro país, pone en evidencia el hecho de que este adolescente "aislado" tiene menos posibilidades de recibir ayuda de quienes evidentemente mas considera; sus pares y su entorno más significativo. Esto tendría inmediatas consecuencias, toda vez que esta comprobado la eficacia de los mecanismos de control informal en casos de delincuencia juvenil y que además en la medida de que un joven se siente parte de una comunidad puede revertir de mejor forma sus conductas antisociales.

\subsection{El Rol de las Redes Sociales en el Proceso de Inclusión de Adolescentes I nfractores}

Como hemos referido con anterioridad seguimos las observaciones de Robles con referencia a los procesos mixtos de inclusión, denominados exclusión en la inclusión, quien lo define como "la inclusión que no es...", cuando no se tiene acceso a las redes de favores, influencias y reciprocidades de conveniencia (Schulze 1995 en: Robles 2005), es decir, cuando no se puede acceder a recursos existentes en otras redes distintas a la que podría considerarse como la más cercana o propia. Afirmamos que esta es la situación de los adolescentes infractores de ley. En este sentido favorecer ciertas características de las redes de estos menores serán vitales para facilitar su inclusión social.

Fue posible observar en la investigación como características de heterogeneidad, dispersión, y por sobretodo vínculos débiles (aquellos menos próximos de menor intensidad relacional), se planteaban como factores de protección frente a conductas de riesgo en general. Ya que este aumento en lo que podríamos denominar un índice de variabilidad de los vínculos presentes en la red de un Ego, tendría un valor particular en el establecimiento de puentes que conecten con distintos círculos sociales.

\section{Relaciones Débiles}

El modelo de "la fuerza de las relaciones débiles" de Mark Granovetter, plantea la posibilidad de establecer puentes que conecten -en este caso a adolescentes infractores- con círculos sociales distintos a los mas próximos y contingentes. Garrido lo expresa de la siguiente manera "si consideramos un Ego con su correspondiente círculo densamente relacionado y con algunos conocidos (relaciones débiles) que, a su vez, tienen sus propios círculos sociales diferentes al de Ego, las relaciones débiles de Ego con sus conocidos se convierten en puentes locales de vinculación". Fue posible 
observar estos puentes y su eficacia durante el estudio, estos puentes aportan todo tipo de recursos a los adolescentes, desde información valiosa hasta recursos económicos para solventar emergencias o desafíos que el propio adolescente se plantee en la construcción de su propia biografía. "Los individuos que mantengan pocas relaciones débiles" - continúa Garrido- estarán confinados a las opiniones y actitudes de su círculo social y no se hallarán integrados en el sistema en su conjunto". En este caso particular la propuesta sería favorecer a inclusión a través de enriquecer la "cartera" de contactos débiles de los menores infractores.

Creemos que en la medida de que la distancia que existe entre los adolescentes de sectores empobrecidos y los sistemas funcionales que deben prestarles servicios (educación, salud, etc.) se mantengan como hasta la fecha, las condiciones de exclusión tenderán a agravarse. Es por esto que consideramos pertinente y necesario salir de la mirada individualista de intervención para incluir estrategias del tipo aquí propuesto.

\subsection{El rol de resistencia que la red familiar ocupa en contextos socio- económicos deprivados.}

Usamos la palabra resistencia siguiendo a modo de analogía la función que una resistencia eléctrica tiene en un circuito, esto es, la de reguladora de la cantidad de energía que circule por un sistema. Esta es una característica que ciertos elementos presentan, ya que en vez de conducir electricidad la transforman en calor.

La red familiar se presenta en el caso de los adolescentes no-infractores haciendo las veces de resistencia, toda vez que impide que el circuito familiar se "queme" por la exposición a factores de riesgo que estresan los procesos en su interior. Situaciones descritas en esta investigación como la progresiva individuación, devuelven sobre la red familiar el peso de la responsabilidad frente a los resultados últimos de la cotidianidad; manutención de los hijos, educación, inserción laboral, problemas de salud, habitabilidad, consumo de bienes o conectividad con los servicios sociales, todas serán situaciones que prioritariamente aparece resolviendo la familia. Ahora bien, de lo anterior no debiera seguirse la idea de que la calidad de los servicios disponibles en nuestro país sea tal que los individuos estén absolutamente echados a su suerte, sin embargo parece evidente que el diferencial producido entre la oferta estatal y las reales necesidad subjetivas de los individuos es pagado por la red familiar.

De lo anterior se derivan algunas reflexiones:

- ¿Cuál es la vida útil de tales resistencias?

- ¿Cómo podemos habilitar a las familias para que presenten y mantengan esa característica?, ¿Será pertinente hacerlo?

- ¿Será posible determinar el desgaste y el daño que se le provoca a la estructura familiar ejercer este tipo de rol?

- ¿Cómo se expresará ese daño?

- En términos económicos. ¿Será rentable para las políticas públicas jugar la carta de la co-responsabilidad con todos los ciudadanos, no presentando estrategias diferidas para temas y poblaciones de alta complejidad?

Esperamos estas reflexiones funcionen como posibles líneas de análisis e investigación.

\subsection{Sugerencias}




\section{Soportes reticulares}

Según lo anteriormente argumentado parecería necesario revisar la excesiva centralidad en el individuo que muestran las estrategias de intervención contenidas en la Ley de Responsabilidad Penal Adolescente. Para incorporar puntos de vista sino sistémicos si de relaciones. Para ello parece pertinente dar mayor énfasis a las intervenciones de tipo grupal, como una forma de ofrecer mayores soportes reticulares de la biografía individual, pensados en los pares, los que demostraron tener mutua ascendencia en los patrones decisionales y conductuales de los adolescentes. Medidas de intervención que contengan y observen la dimensión relacional de la problemática y favorezcan la inclusión de estos jóvenes no mediante las expectativas de coordinación sistémica normativa sino más bien mediante la conexión de círculos sociales distantes. RM

\section{Bibliografía}

Aebi, M. 2003. Familia Disociada y Delincuencia: El caso Suizo en los años 90". Revista Electrónica de Ciencia Penal y Criminología.

Banco Interamericano de Desarrollo. Governance Matters' III: Indicadores de Gobernabilidad 1996-2002.

Disponible en: http://www.bancomundial.org [Fecha de acceso: Febrero 4, 2009]

Bandura, A. 1987 Teoría del aprendizaje social, (trad. Ángel Rivière), 3ạ ed. Espasa Calpe, Madrid, pág. 10.

Bazemore, Gordon. Erbe Carsten. 2003 Operationalizing the Community Variable in Offender Reintegration: Theory and Practice for Developing Intervention Social Capital. Violence and Juvenile J ustice J ournal. 1; 246

Benvenutti, Patrizia. Violencia juvenil y Delincuencia en la región de Latinoamérica. Tesina para la London School of Economics. London School of Economics. 2003.

Beck, Ulrich. "Risk Society, Cosmopolitan Moment".

Disponible en: http://www.harvardpress.com/cosmoplitanmoment.html [Fecha de Acceso: Marzo 19, 2009] Lectura en la Universidad de Harvard. Noviembre 2008.

Beck, Ulrich. 1992 Risk Society: Towards a New Modernity. New Delhi: Sage.

Beck, Ulrich La sociedad de Riesgo. Hacia una nueva modernidad. Ed. Paidos, Barcelona, 1998.

Carlos Vázquez González 2003. Delincuencia juvenil. Consideraciones penales y criminologías, Colex, Madrid.

Cooper, Doris. 2001. Criminología y delincuencia femenina en Chile. Ed. LOM.

Corsi, G. 1996. Glosario sobre la teoría social de Niklas Luhmann. México, U. Iberoamericana, pp. 54-56.

Dammert, Lucía. 2005. “Violencia Criminal y Seguridad Ciudadana en Chile" en Serie 
Políticas Sociales. Editado por CEPAL, Chile.

Dyson Diane Key factors associated with youth delinquency disponible en: http://buildingstrongcommunities. wordpress.com/2009/05/31/key-factors-associatedwith-youth-delinquency/

ECLAC. Social Panorama in Latin America. 2008.

Espinoza, Vicente. 1995. "Redes Sociales y Superación de la Pobreza". Revista de Trabajo Social 66. Santiago. PUC. En aula virtual

Escobar, Santiago, Muniz, Jacqueline, Sanseviero, Rafael, Saín, Marcelo, Zacchi, José Marcelo. 2004. La Seguridad Ciudadana Como Política de Estado. Fundación Friedrich Ebert Stistung. Prosur

Garrido, Francisco. 2001. El Análisis de Redes en el Desarrollo Local. Facultad de Ciencias Política y Sociología. Universidad Complutense de Madrid.

Giddens, Anthony. Diversity and Trust. Artículo aparecido en el periódico ingles The Guardian 2006.

Disponible en: http://www. prospect-magazine.co.uk/article_details. php?id=9886. [Fecha de Acceso: Febrero 17, 2009]

Giddens Anthony. 1990. The Consecuences of Modernity. Polity Press.

Gobierno de Chile, Ministerio del Interior. 2004. Políticas de Seguridad Ciudadana en Europa y América Latina, Lecciones y Desafíos. Serie Libros. Universidad Alberto Hurtado.

Gómez, A. 2006. Tendencias actuales de la Ciencia Criminológica y su contextualización en Cuba.

Disponible en: http://www.uh.cu/.

Greven. P. 2006. "Proteger Niños". Las Raíces Religiosas del Castigo e Impacto Psicológico del Abuso Físico. publicado Alfred A. Knopf, Inc. 1990.

Gryspan, Maira. Ponencia de la Directora Regional del PNUD en el Seminario Medios y Seguridad Ciudadana, Montevideo 2008.

Disponible en http://www.youtube.com/55f644.0s89e47rftdsl [Fecha de acceso: Marzo 21, 2009]

Hernández Sampieri, 2003. Fundamentos de metodología de la investigación. McGrawHill.

Kierkegaard, Søren. 2001. "La Época Presente”, Editorial Universitaria, Pág. 59.

Licha, Isabel. 2001. Las redes de políticas sociales: Un desafío para la gerencia social. Ponencia $V$ Congreso Internacional Sobre la reforma del Estado y la administración pública CLAD.

Luhmann, Niklas. 1992 "Sociología del Riesgo". Universidad I beroamericana, México. 
Mascareño, Aldo. 2005 "La imposibilidad de la Igualdad por la Vía Educativa". Informe 513 Asuntos Públicos.org

Mettifogo, Decio. Sepúlveda, Rodrigo. 2005. Trayectorias de Vida de Jóvenes Infractores de Ley. Serie Estudios. Centro de Estudios en Seguridad Ciudadana. Universidad de Chile.

Moser, Caroline. Lister Sarah. 1999. "Violence and Social Capital". World Bank Latin America and Caribbean Region Environmentally and Socially Sustainable Development SMIJ

Ortí, A. 1997. La confrontación de modelos y niveles epistemológicos en la génesis e historia de la Investigación social. En: Delgado, Juan Manuel y Gutiérrez, Juan (Editores). Métodos y técnicas cualitativas de investigación en Ciencias Sociales. Madrid, Síntesis.

Pardinas, F. Metodología y técnicas de investigación en ciencias sociales: introducción elemental. 1972. Editorial S. XXI

Pizarro, Julio. 2009. Seguridad Ciudadana y Calidad de Vida. Centro de Investigaciones Avance.

Obtenido desde: http://www.centroavance.cl/pizarro [Fecha de acceso: Diciembre17, 2008]

Rice, F. 1997. Child and Adolescent Development. Prentice Hall, Pearson Educación.

Robles, Fernando. 2006. La modernidad “irresponsable": la comunicación de la "irresponsabilidad organizada" en sociedades de riesgo. Revista MAD $N^{\circ} 13$ departamento de antropología. Universidad de Chile.

Robles, F. 2005. Contramodernidad y Desigualdad Social: Individualización e individuación, inclusión/exclusión y construcción de identidad. La necesidad de una sociología de la exclusión. Revista MAD N¹3 departamento de antropología. Universidad de Chile.

Ruiz, José Ignacio. 2003. Metodología de la Investigación Cualitativa. 3ạ Ed. España, Universidad de Deusto.

Torres, Emilio. De la Puente, Patricio. 2006. Seguridad Ciudadana y Sistemas Sociales Autorreferentes en el Contexto de la Sociedad Compleja. Revista De Opinión Jurídica.

Wolf, M. 2001. Investigación en Comunicación de Masas, Paidos, Buenos Aires.

\section{Notas}

1 El presente artículo se basa en los resultados obtenidos en la investigación “Redes Sociales y Posibilidades de Inclusión de Adolescentes Infractores de Ley" presentada para obtener el grado de Magíster en Antropología y Desarrollo de la Universidad de Chile. Dirigida por el profesor Vicente Espinoza E, con una beca.

2 Es interesante en este sentido observar la similitud de las reflexiones actuales y las históricas en torno al fenómeno de la delincuencia juvenil. El periódico Times (1862) en la Inglaterra victoriana publicaba en referencia fenómeno de los niños ladrones y el "Garotting": "Nuestras calles no son tan seguras como lo eran 
Articulación de miradas para una aproximación a la inclusión social de adolescentes infractores de ley.

Teorías de redes sociales y de sistemas sociales

en los tiempos de nuestros abuelos. Hemos regresado a un estado intolerable de los asuntos". Al respecto ver: http://www.timesonline.co.uk/tol/comment/columnists/article4368413.ece

3 Representada en la opinión pública y en el acto eleccionario.

4 Junto con la Pobreza y el Trabajo.

5 Debido a las particularidades que la adolescencia como etapa de desarrollo presenta, en tanto sujeto en construcción, que aún no han llegado a la total madurez psicológica y afectiva.

6 "Hacer efectiva la responsabilidad de los adolescentes por los hechos delictivos que comentan, de tal manera que la sanción forme parte de una intervención socioeducativa amplia y orientada a la plena integración social" Art. 20 Ley 20.084 de Responsabilidad Penal Adolescente.

7 Gobierno de Chile Ministerio de Justicia.

8 Base de datos Carabineros de Chile. 2003

9 Mascareño, A (2005) La imposibilidad de la igualdad por la vía educativa. Bibliografía curso Diferenciación Funcional en América Latina.

Disponible en Internet en: www.asuntospublicos.org/descargaPDF.php?id=3052\&url=513.pdf

10 Marcelo Aebi, 2003. "Familia Disociada y Delincuencia: El caso Suizo en los años 90". Revista Electrónica de Ciencia Penal y Criminología.

11 Aquellas relaciones unidas por lazos debiles son aquellas cuya vinculación entre los actores es poco mas que el simple conocimiento, formando redes de baja densidad, caracterizadas por la asimetría y la heterogeneidad.

12 Los individuos con los cuales comparten la habitación y la economía domestica. 